\title{
Artículo
}

\section{Troponinas cardiacas: \\ Correlación entre el valor real y la injuria miocárdica}

\section{Cardiac troponins: Correlation between real value and the myocardial injury}

Dr.Wadgner Antonio Vargas Fonseca

Médico general, egresado de la Universidad de Costa Rica

Dr.Erick JoséRojas Mora

Médico general, egresado de la Universidad Latina de Costa Rica

\section{Dra.Daniela Maria Navas Gámez}

Médica general, egresada de/la/Universidad Latina de Costa Rica

Correspondencia: wadgvargas@gmail.com

\section{Resumen}

Las troponinas son parte de estructuras miocárdicas que se liberan durante el proceso infarction de daño tisular generado por los diferentes tipos de infarto o de patologías que pueden llegar a producir un efecto deletéreo en la estructura muscular. Han formado parte de los diferentes métodos de diagnóstico e incluso de seguimiento, aunque el nivel de concentración definido no ha sido estandarizado. Además, se intenta hacer una correlación entre las diferentes isoformas y su 
utilidad a corto y largo plazo. Es por esto que es importante mantener la perspectiva de que los marcadores de daño miocárdico orientan al clínico en la objetividad diagnóstica.

\section{Palabras clave:}

Troponina, Isquemia, Infarto miocárdico

\section{Keywords}

Troponin, Ischemia, Myocardial infarction

\section{Abstract}

Troponins are part of myocardial structures that can be found by different assays during the process of tissue damage by different types of infarctions or pathologies that can produce a deleterious effect in the muscular structure.

They have been part of various diagnosis methods including tracing, even though the standard level hasn't been stablished. Furthermore, it is trying to make a correlation between the different isoforms and its utility in short and long time. That is the reason because it's important to keep the perspective that these markers of myocardial damage address the clinicians in the objective diagnosis.

\section{Introducción}

Las troponinas cardiacas son parte de las proteínas estructurales del miocardio y están unidas a nivel del sarcómero, tan solo un 5\% de estas se encuentran de forma libre a nivel sanguíneo(1,2). En condiciones de isquemia, la troponina ubicada a nivel citoplasmático se libera primero, posteriormente conforme se desarrolla un proceso de degradación de miofibrillas. El nivel de troponina se estabiliza en un valor anormal por encima del corte establecido, lo que permite una correcta cuantificación de la misma tenido una alta sensibilidad(1).
Las troponinas cardiacas han sido importantes para la estratificación del riesgo tanto en síndrome coronario agudo (SCA) como en el daño cardiaco no relacionado a isquemia. Tanto la troponina I (cTnI) como la troponina $\mathrm{T}(\mathrm{c} \mathrm{TnT})$ han reemplazado el uso de la creatinin kinasa (CK) debido a una mayor especificidad del tejido miocárdico, por lo que se ha propuesto en diferentes estudios. El uso de estos marcadores como predictores de enfermedad miocárdica, aunque en muchos casos no hay consenso del nivel de corte de los mismos(2-4). Algunos estudios mencionan que tan solo 1 nivel por encima del percentil 99 obliga al clínico a estudiar al paciente en busca de algún hallazgo que correlacione con infarto al miocardio.

Ciertos hallazgos por debajo del percentil podrían no estar relaciones con un amplio margen de enfermedad cardiaca, sino incluso en periodos de intervención temprana o bien en otras patologías diferentes al infarto de miocardio: hipertrofia ventricular, angina estable, falla cardiaca, o incluso niveles de normalidad (nivel $\leq 5 \mathrm{ng} / \mathrm{L})(1,4,5)$.

Este trabajo pretende realizar una revisión de los estudios más recientes en relación al uso de las troponinas con el fin de determinar si existe o no un corte real que permita una correlación exacta entre el nivel de injuria cardiaca y el hallazgo de laboratorio.

\section{Marcadores cardiacos y valores predichos}

La prueba para detección de cTnT utiliza una porción de ligando de fragmento de antígeno (FAB) de 2 anticuerpos monoclonales de ratón específicos para cTnT (MAbs). Se realiza una quimera biosintética con el uso de fragmentos de $\mathrm{C} 1$ de una $\mathrm{IgG}$, que se dirigen de forma directa contra el epitopo de la región central de 
la cTnT humana en los residuos de aminoácidos 121-131(3,6). Las diferentes pruebas existentes se han calibrando conforme a la liberación de la cTnT recombinante humana producida por medio de cultivos celulares de Escherichia coli, $\mathrm{y}$ es importante tener en cuenta que las generaciones varían en el valor del límite superior y con ello el valor diagnóstico. En este caso, un estudio con una prueba de alta sensibilidad tendrá un corte de $10 \mathrm{ng} / \mathrm{L}$, mientras que con una prueba convencional será de $30 \mathrm{ng} / \mathrm{L}$, teniendo un impacto en la sensibilidad y especificidad de la prueba.

En el caso de la prueba de detección convencional, se genera un área de espectro donde los valores que se encuentran entre $10 \mathrm{y}$ 30ng/L, pueden ser o no considerados patológicos dependiendo de la clínica que se presente en el paciente y de la severidad o incluso aumento de la misma en los ensayos desarrollados(1-3,7).

En un pequeño ensayo retrospectivo descrito por McCarthy et al menciona que pequeñas elevaciones en el marcador cardiaco, que se ubiquen dentro del área de espectro, pueden generar en un periodo de 5 años, una irreversibilidad del valor encontrado por encima del límite superior, tanto para la prueba de alta sensibilidad como para la convencional(1).

Lo que ha generado que muchos investigadores y profesionales del campo clínico, lleven a cabo estudios más centrados en las pruebas de valor predictivo positivo para el área de espectro no especificada.

Sin embargo, se conoce de factores que impulsan y desarrollan con mayor facilidad el proceso de injuria cardiaca al punto de generar zonas de necrosis que permitan la liberación de las troponinas de los sarcómeros: edad, hipertensión, angina estable, hiperlipidemia, enfermedad renal, diabetes, enfermedad obstructiva crónica, entre otras, modificando el valor de riesgo basal de un $35 \%$ a un $89 \%(1,4,5,8)$. Por otro lado, autores como Lee et al, mencionan que la elevación de las troponinas a nivel sanguíneo no siempre corresponde a una injuria cardiaca mayor o a un padecimiento cardiaco que lleve a un desarrollo fortuito o alejado de los hallazgos comunes dependiendo del tipo de paciente, y que, a su vez (principalmente determinado por la edad) el nivel de troponina no correlaciona directamente con el padecimiento en proceso. Se reportan casos de troponinas dentro del área de espectro en paciente mayores de 70 años con un infarto miocárdico tipo en proceso, lo cual no es directamente proporcional con lo que se debería corresponder $(5,8-10)$.

En el caso de la cTnI, esta tiene epítopos diferentes que pueden ser identificados mediante múltiples formas, ya que tiene una estructura un poco más maleable a la concentración de calcio, así como permite identificarse en las porciones de actina de la miofibrilla y no necesariamente con la destrucción del miocito. Sin embargo, esto ha desarrollo múltiples isoformas de antígeno que han disipado la probabilidad de encontrar un método estándar de detección.

Además, la cTnI tiene la desventaja de formar isómeros con otras isoformas de troponinas, haciendo que la sensibilidad aumente pero no bien su especificidad(3,6). Además, se ve mayormente elevada en pacientes con enfermedades no relacionadas de forma directa al infarto agudo al miocardio, por ejemplo insuficiencia cardiaca en estadios tempranos $(7,8,11)$. 


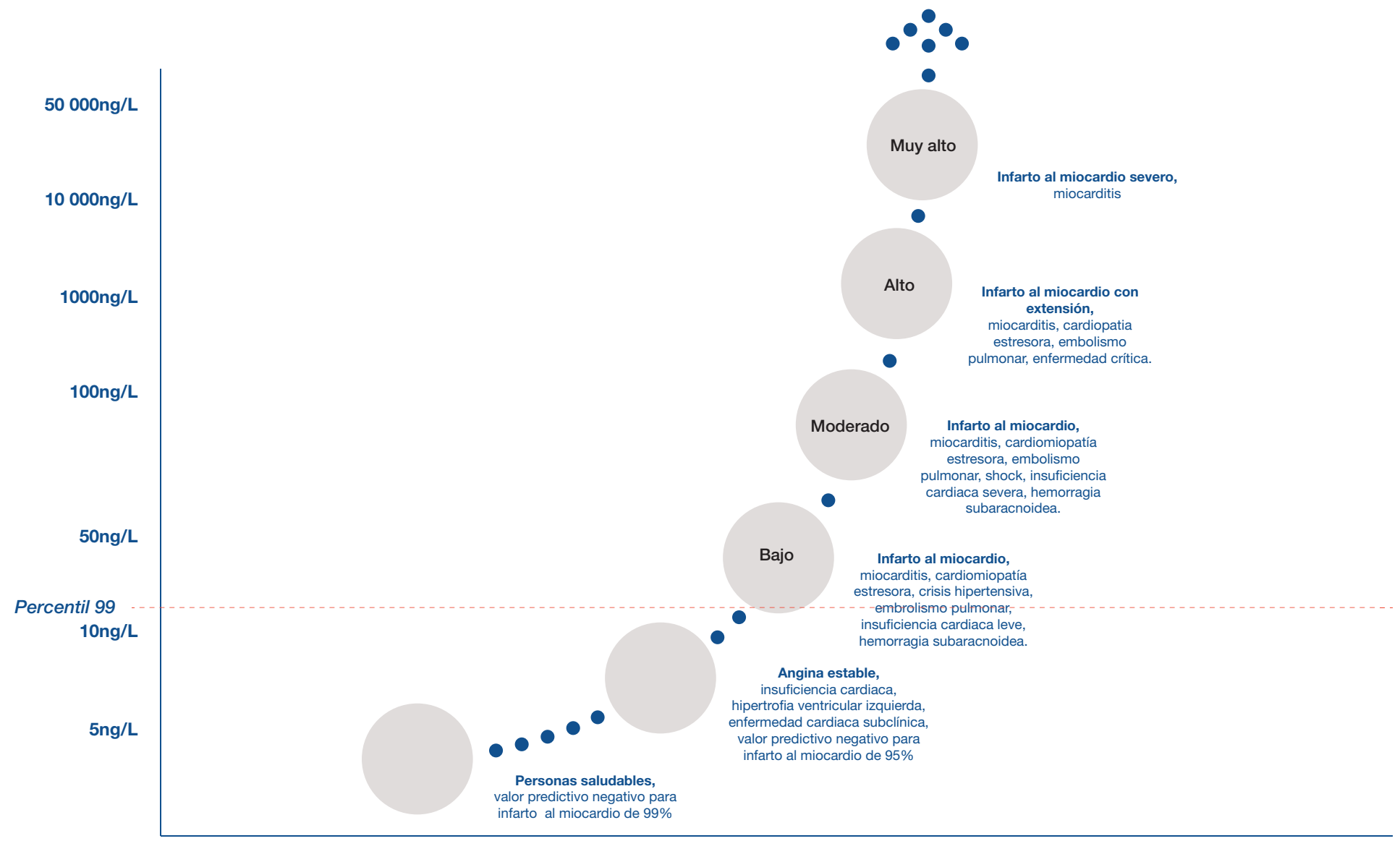

Figura 1. Valor diagnóstico del nivel de troponina con su correlación clínica (7).

En algunos estudios se menciona que el uso de la cTnI puede darse como un parámetro con valor predictivo positivo alto más allá de formar parte de los hallazgos de laboratorios propios de una emergencia cardiaca inminente, es decir, la elevación de la cTnI puede dar lugar a especulaciones sobre el pronóstico del paciente en un periodo determinado(11,12).

Por otro lado, se debe tomar en cuenta, que estas pruebas, debido a la alta variabilidad y baja estandarización, pueden tener un margen de error de aproximadamente $3.4 \mathrm{ng} / \mathrm{L}$ incluso en concentraciones que no deberían preocupar al clínico (un valor <10ng/L)(11). De ahí que, algunos autores como Sandoval et al mencionen que, aunque el nivel de valor predictivo positivo alcanza hasta un $98 \%$ con un aumento del $20 \%$ en un periodo de 2 años, la especificidad ante un evento agudo desciende hasta un $28-65 \%$.

Aportan que este tipo de pruebas combinadas con la cTnT, podrían entonces favorecer la probabilidad de diagnosticar de forma temprana los infartos cardiacos y prevenir el avance de los diferentes tipo de injurias cardiacas(12).

\section{Tipos de infarto y la utilidad de las troponinas}

Se debe tener claro que el infarto agudo al miocardio es aquel que produce un aumento o descenso dinámico del nivel de troponinas, ya sea atribuible a una causa cardiovascular o no, y el daño crónico al miocardio es aquel que tiene una elevación constante de la concentración sérica de troponinas por algunas de las mismas causas descritas. 
Lo que los diferencia, es ese proceso dinámico en la concentración del marcador de daño cardiaco(1).

Por otro lado, los marcadores tienen una utilidad destinada dependiendo del tipo de infarto que se presente en el paciente. La mayor cantidad de estudios se han destinado a investigar sobre el impacto y la aparición de las troponinas cuando el subtipo de infarto al miocardio se debe a un desorden de oxigenación y demanda, dando resultados ampliamente favorables para la detección de la troponina, debido al alto daño generado en las fibras del miocito durante el proceso de daño tisular(12). Sin embargo, algunos otros se han enfocado en el subtipo 1, con mayor énfasis en la utilidad de la cTnI como factor predictivo incluso en los periodos previos a desarrollar el evento (tal como se explicó antes de la utilidad de la cTnI) $(6,13)$.

Tabla 1. Definición de los subtipos de infarto al miocardio(1).

\section{Tipo de infarto al miocardio}

\begin{tabular}{|c|c|}
\hline \multicolumn{2}{|c|}{$\begin{array}{l}\text { TIPO DE INFARTO } \\
\text { AL MIOCARDIO }\end{array}$} \\
\hline & $\begin{array}{l}\text { Infarto miocárdico causado por } \\
\text { ruptura, ulceración o disección de } \\
\text { placa }\end{array}$ \\
\hline 2 & $\begin{array}{l}\text { Infarto micárdico atribuible a } \\
\text { desorden en oxigenación y } \\
\text { demanda }\end{array}$ \\
\hline 3 & $\begin{array}{l}\text { Muerte cardiaca aguda con un } \\
\text { mecanismo de causa similar al } \\
\text { infarto miocárdico }\end{array}$ \\
\hline 4 & $\begin{array}{l}\text { Infarto miocárdico asociado a } \\
\text { intervención percutánea o } \\
\text { trombosis de stent }\end{array}$ \\
\hline 5 & $\begin{array}{l}\text { Infarto miocárdico asociado } \\
\text { con cirugía cardiaca }\end{array}$ \\
\hline
\end{tabular}

El autor Blankenberg et al, hace referencia a la forma dinámica de las troponinas en un infarto subtipo 1. El algoritmo generado en su investigación, menciona que puede haber una varianza que no ha sido totalmente estandarizada desde la primera hora hasta $6 \mathrm{~h}$ después de la aparición de la clínica del paciente, mientras que en el caso de los algoritmos destinados para los subtipo 2 puede alcanzarse una estabilidad de la concentración de troponina en un periodo no mayor a 3 horas $(7,14)$, aunque es enfático en que la estabilidad completa se alcanza únicamente en patologías crónicas establecidas como la miocardiopatía.

El nivel de troponina permite la estratificación del riesgo que tenga el paciente no solo para el evento agudo o crónico, sino además para el tipo de tratamiento que tenga posterior al desarrollo de un evento de injuria cardiaca.

\section{Impacto y pronóstico}

El uso del nivel de concentración de troponina como un medidor de seguimiento, se ha llevado por medio de varios estudios. Donde se ha visto que la diferencia en el nivel del predictor genera un resultado estadísticamente significativo con el uso de medicamentos a nivel de prevención secundaria $(10,15)$.

En un estudio de seguimiento de 172 pacientes divididos por grupos de edades, se observó que el uso de las troponinas como marcadores de atención primaria han dado un impacto de resolución de hasta un $98 \%$, con disminución de la mortalidad en un periodo comprendido de 30 días hasta 10 años, y descenso en la escala HEART de hasta 4 puntos en casos donde se logra hacer intervención oportuna, con pruebas de alta sensibilidad de troponinas(16).

Sin embargo, cabe destacar que esto eleva los costos de la medicina limitando el acceso a ciertos servicios de salud(16), así como interpone la necesidad de tener personal 
capacitado para hacer un análisis clínico correcto entre el nivel reportado y la severidad del cuadro del paciente. Es por estas razones que el proceso de elección de los estudios se ha limitado a las diferentes poblaciones en casos en los que sea necesario.

No obstante, cabe descartar, que, aunque algunos estudios no sean concluyentes, siempre prevalecerá el aspecto clínico-diagnóstico por encima de los resultados de laboratorio.

\section{Conclusión}

Es importante tener en cuenta que el nivel de concentración de troponina varía con la generación de la prueba que se realice, así como el contexto del paciente en el cual se ejecute la prueba. El desarrollo de la injuria y su evolución natural darán valores elevados de las mismas en proporción directa al hallazgo descrito.

La ausencia en el consenso de estandarización respecto al nivel de corte para generar alarma en el proceso diagnóstico, no interrumpe el correcto abordaje del paciente. Obliga al clínico a avanzar de forma cautelosa dependiendo de la experiencia y de la capacidad de procesar los aspectos objetivos y la relación entre las variables. Si bien los estudios hablan del uso de troponinas a nivel primario, en nuestro país es limitado por el recurso económico de los centros de salud.

\section{Referencias}

1. McCarthy CP, Raber I, Chapman AR, Sandoval Y, Apple FS, Mills NL, et al. Myocardial Injury in the Era of

High-Sensitivity Cardiac Troponin Assays: A Practical Approach for Clinicians. JAMA Cardiol. 2019;4(10):1034-42.

2. Twerenbold R, Jaffe A, Reichlin T, Reiter M, Mueller C. High-sensitive troponin $\mathrm{T}$ measurements: What do we gain and what are the challenges? Eur Heart J.

2012;33(5):579-86.

3. Apple FS, Sandoval Y, Jaffe AS, Ordonez-Llanos J. Cardiac troponin assays: Guide to understanding analytical characteristics and their impact on clinical care. Clin Chem. 2017;63(1):73-81.

4. Sarkisian L, Saaby L, Poulsen TS, Gerke O, Jangaard N, Hosbond S, et al. Clinical Characteristics and Outcomes of Patients with Myocardial Infarction, Myocardial Injury, and Nonelevated Troponins. Am J Med [Internet].

2016;129(4):446.e5-446.e21. Available from: http://dx.doi.org/10.1016/j.amjmed.2015.11.006

5. Lee KK, Noaman A, Vaswani A, Gibbins M, Griffiths M, Chapman AR, et al. Prevalence, Determinants, and Clinical Associations of High-Sensitivity Cardiac Troponin in Patients Attending Emergency Departments. Am J Med [Internet]. 2019;132(1):110.e8-110.e21. Available from: https://doi.org/10.1016/j.amjmed.2018.10.002

6. Oda T, Yanagisawa $H$, Wakabayashi $T$. Cryo-EM structures of cardiac thin filaments reveal the 3D architecture of troponin. J Struct Biol [Internet]. 2020;20(1):30004-6. Available from: https://doi.org/10.1016/j.jsb.2020.107450 
7. Januzzi JL, Mahler SA, Christenson RH, Rymer J, Newby LK, Body R, et al. Recommendations for Institutions Transitioning to High-Sensitivity Troponin Testing: JACC Scientific Expert Panel. J Am Coll Cardiol. 2019;73(9):1059-77.

8. Gibson CM, Morrow DA. Elevated Cardiac Troponin in the Absence of Acute Coronary Syndromes. Card Intensive Care. 2019;196-202.

9. Sandoval Y, Thygesen K. Myocardial infarction type 2 and myocardial injury. Clin Chem. 2017;63(1):101-7.

10. Chapman AR, Shah ASV, Lee KK, Anand A, Francis O, Adamson P, et al. Long-term outcomes in patients with type 2 myocardial infarction and myocardial injury. Circulation. 2018;137(12):1236-45.

11. Kavsak PA, Worster A, Hill SA, MacRae AR, Jaffe AS. Analytical comparison of three different versions of a high-sensitivity cardiac troponin I assay over 10 years. Clin Chim Acta. 2017;475(October):51-5.

12. Sandoval Y, Thordsen SE, Carlson MD, Johnson BK, Smith SW, Bruen CA, et al. Type 1 and 2 Myocardial Infarction and Myocardial Injury: Clinical Transition to High-Sensitivity Cardiac Troponin I. Am J Med [Internet]. 2017;130(12):1431-1439.e4. Available from:

http://dx.doi.org/10.1016/j.amjmed.2017.05.049

13. Goldstein SA, Newby LK, Cyr DD, Neely M, Lüscher TF, Brown EB, et al. Relationship Between Peak Troponin Values and Long-Term Ischemic Events Among Medically Managed Patients With Acute Coronary Syndromes. J Am Heart Assoc. 2017;6(4).
14. Blankenberg S, Wittlinger T, Nowak B, Rupprecht HJ. Troponine als Biomarker bei Myokardschaden und Myokardinfarkt. Springer Medizin Verlag GmbH. 2019;44(1):4-9.

15. Mokhtari A, Borna C, Gilje $\mathrm{P}$, Tydén $\mathrm{P}$, Lindahl B, Nilsson HJ, et al. A 1-h Combination Algorithm Allows Fast Rule-Out and Rule-In of Major Adverse Cardiac Events. J Am Coll Cardiol. 2016;67(13):1531-40.

16. Davis A, Chiu J, Lau SK, Kok YJ, Wu JYH. Efficacy of implementation of a chest pain center at a community hospital. Crit Pathw Cardiol. 2017;16(4):135-41. 